\title{
Soluble triggering receptor expressed on myeloid cells-1 as a useful biomarker for diagnosing ventilator-associated pneumonia after congenital cardiac surgery in children
}

\author{
CHUNXIANG LI ${ }^{1}$, LIMIN ZHU ${ }^{1}$, XIAOLEI GONG ${ }^{1}$, ZHUOMING XU $^{1}$, \\ YUJIE LIU ${ }^{1}$, MINGJIE ZHANG ${ }^{1}$ and QING CAO $^{2}$ \\ Departments of ${ }^{1}$ Cardiac Intensive Care Unit and ${ }^{2}$ Infectious Disease, Shanghai Children's Medical Center, \\ Shanghai Jiaotong University School of Medicine, Shanghai 200127, P.R. China
}

Received April 24, 2018; Accepted October 4, 2018

DOI: $10.3892 /$ etm.2018.6905

\begin{abstract}
The present study aimed to assess the usefulness of soluble triggering receptor expressed on myeloid cells 1 (sTREM-1) in the diagnosis of ventilator-associated pneumonia (VAP) in paediatric patients with congenital heart disease (CHD) following cardiac surgery. The current prospective study enrolled 48 patients with congenital heart diseases who were suspected of having VAP; these patients were undergoing cardiac surgery between August 2016 and October 2017 in the Cardiac Intensive Care Unit of Shanghai Children's Medical Center (Shanghai, China). A total of 31 patients were diagnosed with VAP using a polymerase chain reaction (PCR) assay, while 17 patients without VAP were designated as the Non-VAP group. A bronchoscopy was performed and samples were collected for measurement on the day that VAP was diagnosed. The sTREM-1 levels were measured in bronchoalveolar lavage fluid (BALF) and exhaled ventilator condensate (EVC). BALF specimens were also sent to the microbiology laboratory for PCR assays and quantitative culturing. The positive detection rate of bacteria using the PCR assay and traditional culture was $64.6 \%(31 / 48)$ and $39.6 \%(19 / 48)$. sTREM-1 was significantly higher in the BALF $(146.21 \mathrm{pg} / \mathrm{ml}$ vs. $118.06 \mathrm{pg} / \mathrm{ml})$ and EVC (125.29 pg/ml vs. $120.48 \mathrm{pg} / \mathrm{ml})$ of patients with VAP demonstrated compared with the patients without VAP. The findings suggest that the detection of sTREM-1 in BALF and EVC samples may be useful for the diagnosis of VAP following heart surgery in children.
\end{abstract}

Correspondence to: Dr Limin Zhu, Department of Cardiac Intensive Care Unit, Shanghai Children's Medical Center, Shanghai Jiaotong University School of Medicine, 1678 Dongfang Road, Shanghai 200127, P.R. China

E-mail: zhulimin121@hotmail.com

Key words: soluble triggering receptor expressed on myeloid cells, congenital heart disease, ventilator-associated pneumonia, polymerase chain reaction

\section{Introduction}

Ventilator-associated pneumonia (VAP) is a common type of nosocomial infection in children following cardiac surgery $(1,2)$. Transient systemic immune suppression (3) and the prolonged use of mechanical ventilation increase the risk of VAP following cardiac surgery (2). It has been reported that VAP increases the duration of mechanical ventilation, prolonging the hospital stay (4) and even contributing to a $13 \%$ mortality rate (5). However, it is a major challenge to accurately diagnose VAP in patients immediately following cardiac surgery as systemic inflammatory response syndrome is induced by surgical trauma as well as the interaction of the blood with the cardiopulmonary bypass (6), and imaging studies may reveal pulmonary opacities caused by the surgical manipulation, atelectasis or alveolar haemorrhage (4). As a result, accurate pathogen detection to discern advisable treatment and early diagnosis are key to improving the prognosis of patients with VAP following heart surgery.

The gold standard for the diagnostic confirmation of VAP is lung tissue examination and culturing $(7,8)$, however, as an interventional procedure is required to get lung biopsy samples, clinical applicability is limited (8). The triggering receptor expressed on myeloid cells-1 (TREM-1) is a member of the immunoglobulin superfamily, is secreted by neutrophils, macrophages and monocytes, and amplifies the inflammatory response following the exposure of cells to bacteria and fungi (9). A soluble form of TREM-1 (sTREM-1) has been proposed as a novel biomarker, and has been tested for in patients with acute infections with different diagnostic and prognostic results (9-11). Elevated levels of sTREM-1 were identified in the serum, bronchoalveolar lavage fluid (BALF) and the exhaled ventilator condensate (EVC) in patients with VAP $(12,13)$. sTREM-1 in exhaled breath condensate (EBC) and BALF has been demonstrated to be a good diagnostic factor in differentiating patients who have suffered an ischaemic stroke with VAP from those without (14), however the measurement of sTREM-1 in EVC has been demonstrated to be useful for the diagnosis of VAP after cardiac surgery (13). Whether the detection of sTREM-1 in EVC and BALF improves the accuracy of a VAP 
diagnosis following cardiac surgery remains to be explored. Additionally, polymerase chain reaction (PCR) assays allow for rapid molecular testing, as traditional culturing methods may not be sufficiently sensitive to detect even the more common bacterial pathogens and viruses (15-17). Studies using PCR techniques for pathogen detection have involved severe sepsis and bloodstream infections $(17,18)$. A previous study demonstrated that PCR assay rapidly detected pathogens, and was more sensitive and rapid than traditional cultures (19). In the present study, a PCR assay was used to define the pathogens in BALF for patients with suspected VAP, and to evaluate whether the use of sTREM-1 improved the accuracy of VAP diagnosis in children undergoing cardiac surgery.

\section{Materials and methods}

Study design. The current study was a prospective cohort study conducted in the Cardiac Intensive Care Unit (CICU) of Shanghai Children's Medical Center (Shanghai, China) on children with congenital heart disease undergoing cardiac surgery between August 2016 and October 2017. The present study was approved by the Ethics Committee of Shanghai Jiaotong University School of Medicine (Shanghai, China; approval no. SCMCIRB-K2015040) and written informed consent was obtained from the patients' parents.

Diagnosis, treatment and prevention of VAP. Patients with suspected VAP who remained intubated and mechanically ventilated for $\geq 48 \mathrm{~h}$ after surgery were included. VAP was suspected if the patient had a radiographic infiltrate that was novel or progressive, together with clinical findings that were suggestive of infection, including the onset of fever (temperature $\geq 38.3^{\circ} \mathrm{C}$ ) or hypothermia (temperature $\leq 36.5^{\circ} \mathrm{C}$ ), leucocytosis $\left(\geq 10 \times 10^{9} / 1\right.$ or $\left.\leq 4 \times 10^{9} / 1\right)$, purulent sputum and a decline in oxygenation (oxygen saturation $<90 \%$ ). The exclusion criteria were a preoperative diagnosis of pneumonia and/or sepsis. The BALF samples were collected and analysed as described below. Patients with a positive detection of bacteria were determined as the VAP group, whilst the control group was determined as the non-VAP group, patients with a negative detection of bacteria.

The protocol for VAP treatment and prevention followed standard protocols based on the British Thoracic Society guideline for advanced diagnostic and therapeutic flexible bronchoscopy in adults (20). In addition, VAP care bundles for the prevention of VAP were also available (20).

Clinical assessment. The baseline assessment included the evaluation of demographic data (age, sex and weight), medical history, Paediatric Risk of Mortality score (21), Risk Adjustment for Congenital Heart Surgery score (22), modified clinical pulmonary infection score (23), the ratio of partial oxygen to the fraction of inspired oxygen $\left(\mathrm{PaO}_{2} / \mathrm{FiO}_{2}\right)(23)$, cardiopulmonary bypass time, aortic cross clamp time, the level of inflammatory biomarkers procalcitonin and C-reactive protein, the time of intubation, CICU length of stay and hospital length of stay. The levels of procalcitonin in blood plasma were determined using a procalcitonin detecting kit and measured by Getein 1600 Immunofluorescence
Quantitative Analyzer (both Getein Biotech, Inc., Nanjing, China). The C-reactive protein levels in blood were determined using a C-reactive protein detecting kit (Goldsite, Inc., Shenzhen, China) and detected by Astep C Reactive Analyzer (GOLDSITE, Inc.).

Sample processing and measurement. A bronchoscopy was performed on the day when VAP was suspected and BALF and EVC samples were collected for measurement on the same day. The diagnostic flexible bronchoscopy guideline of the British Thoracic Society was also followed (8). To obtain the BALF sample, a total of $9 \mathrm{ml}$ sterile saline was instilled into the middle lobes of the right and left lungs, and was then gently suctioned out. One-third of the BALF sample was centrifuged at $200 \mathrm{x} \mathrm{g}$ for $15 \mathrm{~min}$ at room temperature, and the cell-free supernatants were aliquoted. In addition, EVC samples, the liquid of exhaled gases and vapours collected in a portable condenser, were collected from the trap located in the expiratory limb of the ventilator circuit and $3 \mathrm{ml}$ was required for the measurement on the same day. Part of the BALF and EVC samples were sent to the laboratory immediately following the collection to measure the sTREM-1 protein concentration using the Human TREM-1 Quantikine ELISA kit (cat. no. DTRM10B; R\&D Systems Inc., Minneapolis, MN, USA). The remainder of the BALF samples were analysed by quantitative PCR (qPCR) and microbiological culture using PMseq ${ }^{\mathrm{TM}}$ infection high-throughput gene detection analysis performed by Beijing Genomics Institute (Beijing, China).

Statistical analysis. The statistical analysis was performed using SPSS version 19.0 (IBM Corp., Armonk, NY, USA). Data are expressed as the median (range). The VAP positive and VAP negative data were compared using a Mann-Whitney $\mathrm{U}$ test for equal proportion. The statistical tests performed were two-sided. All the analyses were performed on an intention-to-treat basis and a two-sided $\mathrm{P}<0.05$ indicated that the difference between groups was statistically significant. The association of characteristics with VAP was assessed by Spearman's correlation. A Receiver Operating Characteristic (ROC) curve was constructed to determine the cut-off value of sTREM-1 expression in the EVC for the diagnosis of VAP. The figures were drawn using GraphPad prism version 5.0 (GraphPad Software, Inc., La Jolla, CA, USA) and Medcalc11.4.2 (MedCalc Software bvba, Ostend, Belgium).

\section{Results}

Patient characteristics following clinical assessment. Throughout the study period, 95 children with congenital heart diseases were admitted to the CICU at Shanghai Children's Medical Center following cardiac surgery and 48 of the patients met the inclusion criteria were suspected of having VAP. Among them, 31 were diagnosed with VAP following the positive detection of bacteria using PCR assays and 17 were not. The baseline characteristics of the 48 patients are shown in Table I. The duration of mechanical ventilation, as well as the CICU and hospital length of stay were significantly increased in the VAP group compared with that in the non-VAP control group $(\mathrm{P}<0.01)$. In addition, the $\mathrm{mCPIS}, \mathrm{PO}_{2} / \mathrm{FiO}_{2}(\mathrm{mmHg})$, 
Table I. Patient characteristics of the study groups.

\begin{tabular}{|c|c|c|c|}
\hline Characteristics & Patients with VAP $(\mathrm{n}=31)$ & Patients without VAP $(\mathrm{n}=17)$ & P-value \\
\hline Age (days) & $42(1-2,738)$ & $49(1-3,492)$ & 0.82 \\
\hline Weight (kg) & $3.5(1.9-18.2)$ & $3.6(2.1-23)$ & 0.78 \\
\hline Male sex & $26(54.2 \%)$ & $25(52.1 \%)$ & 0.73 \\
\hline PRISM score & $12(4-27)$ & $11(2-20)$ & 0.25 \\
\hline RACHS-1 score & $4(2-6)$ & $4(2-5)$ & 0.62 \\
\hline mCPIS & $5(3-8)$ & $4(2-8)$ & 0.54 \\
\hline $\mathrm{PO}_{2} / \mathrm{FiO}_{2}(\mathrm{mmHg})$ & $230(135-305)$ & $252(153-320)$ & 0.75 \\
\hline PCT expression level & $5.2(0.9-12)$ & $5(0.8-11)$ & 0.63 \\
\hline CRP expression level & $22(8-89)$ & $25(6-92)$ & 0.35 \\
\hline Use of CPB & $29(93.5 \%)$ & $15(88.2 \%)$ & 0.67 \\
\hline Duration of CPB (min) & $97(0-172)$ & $94(0-156)$ & 0.42 \\
\hline Aortic cross clamp time (min) & $62(0-98)$ & $65(0-109)$ & 0.23 \\
\hline Duration of mechanical ventilation (days) & $7(3-17)$ & $3(1-7)$ & $<0.01$ \\
\hline CICU length of stay (days) & $15(5-30)$ & $7(4-15)$ & $<0.01$ \\
\hline Hospital length of stay (days) & $28(8-210)$ & $13(7-26)$ & $<0.01$ \\
\hline
\end{tabular}

The data are reported as the median (range) or n (\%). PRISM, Paediatric Risk of Mortality; RACHS-1, Risk Adjustment for Congenital Heart Surgery; mCPIS, modified Clinical Pulmonary Infection Score; PCT, procalcitonin; CRP, C-reactive protein; CPB, cardiopulmonary bypass; CICU, Cardiac Intensive Care Unit.
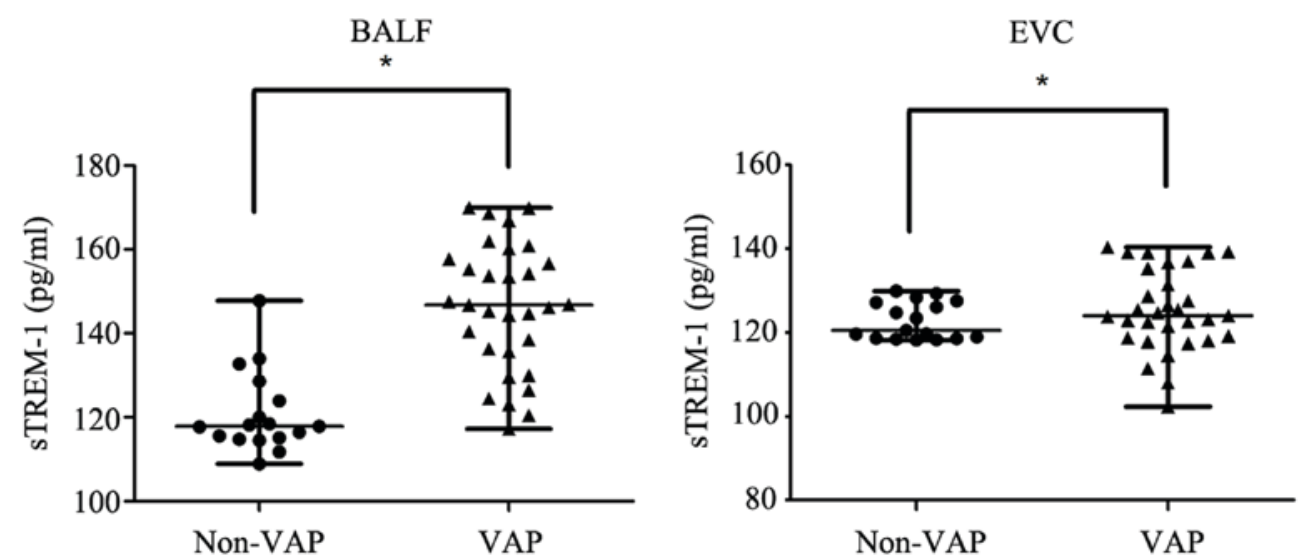

Figure 1. sTREM-1 detection in the BALF and EVC samples of patients with or without VAP. Median sTREM-1 levels in the BALF and EVC samples in 31 patients with and 17 patients without VAP. Each dot represents each individual patient. Data are presented as the median, and range. " P $<0.05$. sTREM-1, soluble triggering receptor expressed on myeloid cells-1; BALF, bronchoalveolar lavage fluid; EVC, exhaled ventilator condensate; VAP, ventilator-associated pneumonia.

PCT and CRP expression level were not significantly different in the VAP group compared with that in the non-VAP control group $(\mathrm{P}>0.05)$.

Bacteria detection by $q$ PCR and microbiological culture. Of the 48 samples, the positive culture rate was $39.6 \%(19 / 48)$. From the culture experiments, a total of 21 pathogens were identified after culturing for $72 \mathrm{~h}$, and $>1$ pathogen was detected in 2 samples (data not shown). Of the 48 samples, 31 (64.6\%) were qPCR positive, confirming the diagnosis of VAP. The qPCR positive samples were defined as the VAP group, whilst the 17 qPCR negative samples were defined as the non-VAP control group. A total of 44 pathogens from 31 samples were detected in just $24 \mathrm{~h}$ of the samples being obtained from patients with suspected VAP. A total of 9 samples had a mixed pathogen infection. Of the 44 pathogens, 8 were Acinetobacter baumannii, 2 were Haemophilus influenza, 4 were Escherichia coli, 3 were Klebsiella pneumonia, 3 were Enterobacter cloacae, 3 were Streptococcus pneumoniae, 6 were Staphylococcus aureus, 2 were Enterococcus faecium, 2 were Stenotrophomonas maltophilia, 2 were Pseudomonas aeruginosa, 3 were Mycoplasma pneumoniae and 6 were Candida albicans. The qPCR results of the BALF samples yielded the best sensitivity and specificity to diagnose VAP, differentiating true infections from inflammation or colonisation.

Detection and comparison of STREM-1 and diagnostic value of VAP. STREM-1 protein concentration was detected in all 48 patients on the day that VAP was suspected. sTREM-1 
protein expression in BALF of the VAP group (median, $146.21 \mathrm{pg} / \mathrm{ml}$; range, $117.26-169.91 \mathrm{pg} / \mathrm{ml}$ ) was significantly higher compared with the Non-VAP group (median, $118.06 \mathrm{pg} / \mathrm{ml}$; range, 108.89-147.76 pg/ml; P<0.05; Fig. 1). The cut-off value of sTREM-1 in BALF on the day of VAP diagnosis was $134.80 \mathrm{pg} / \mathrm{ml}$, which had a sensitivity of $77.5 \%$ and a specificity of $93.8 \%$ for the diagnosis of VAP [area under the ROC curve, 0.91 ; $95 \%$ confidence intervals (CI), 0.83-0.99; Fig. 2]. sTREM-1 protein expression in EVC of the VAP group (median, $125.29 \mathrm{pg} / \mathrm{ml}$; range, $102.31-140.34 \mathrm{pg} / \mathrm{ml}$ ) was significantly higher compared with the Non-VAP group (median, $120.48 \mathrm{pg} / \mathrm{ml}$; range, $118.21-129.91 \mathrm{pg} / \mathrm{ml} ; \mathrm{P}<0.05 ;$ Fig. 1). The cut-off value for sTREM-1 in EVC was $109.75 \mathrm{pg} / \mathrm{ml}$, which had a sensitivity of $93.2 \%$ and a specificity of $76.5 \%$ for the diagnosis of VAP (area under the ROC curve, 0.89; 95\% CI, 0.79-0.98; Fig. 2).

\section{Discussion}

VAP is a major cause of morbidity and mortality following cardiac surgery worldwide, particularly in children (5). Despite advances in diagnostics, it is still challenging to diagnose VAP early, and the aetiology and therapy is empirical (4). As systemic inflammatory response syndrome and VAP have similar characteristics in early development, patients are treated for VAP, which requires treatment with antibiotics, which is excessive and unnecessary for patients with systemic inflammatory response syndrome (24). This excessive and unnecessary use of antibiotics may lead to increased bacterial resistance and increased costs, which highlights the importance of an early and accurate diagnosis of VAP (25). As a result, the current study investigated biological markers of infection, such as sTREM-1, to improve the accuracy of the diagnosis of VAP.

One aspect of the current study was the use of PCR for the diagnosis of VAP. Cultures lack the sensitivity to identify all the bacteria in samples for multiple reasons, including previous antibiotic administration, sampling error and fastidious bacteria $(26,27)$; whereas PCR amplification may supplement cultures when detecting pathogens (16). In the present study, more samples and more pathogens were detected by the PCR assay compared with traditional culturing, and the positive rates were 64.6 and $39.6 \%$, respectively. The results imply that the false negative rate was much higher when the culture method was employed compared with the PCR assay, and the PCR assay was more sensitive for the detection of pathogens compared with traditional culturing.

Several previous studies have reported the diagnostic effects of sTREM-1 protein concentration evaluation in VAP. Yu et al (14) demonstrated that the sTREM-1 concentration was unregulated in EBC and BALF of patients with VAP, allowing the differentiation of patients with ischaemic stroke and VAP. Matsuno and Carlotti (13) demonstrated a similar result for the sTREM-1 concentration in the mBALF of children with and without VAP following congenital heart surgery. In the present study, a significant increase in the sTREM-1 concentration was observed in the BALF sample of the VAP group; the cut-off value was $134.8 \mathrm{pg} / \mathrm{ml}$, with a sensitivity of $77.5 \%$ and a specificity of $93.8 \%$ for the diagnosis of VAP. In addition, the levels of sTREM-1 in the EVC

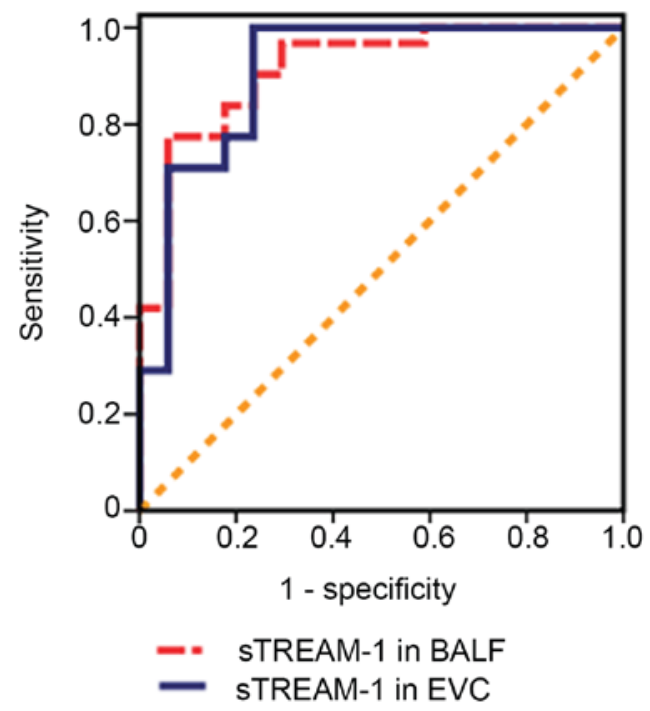

Figure 2. Diagnostic value of sTREM-1. Receiver-operating characteristic curves of sTREM-1 levels in BALF and EVC samples. sTREM-1, soluble triggering receptor expressed on myeloid cells-1; BALF, bronchoalveolar lavage fluid; EVC, exhaled ventilator condensate.

were significantly higher in the VAP group; the cut-off value was $109.75 \mathrm{pg} / \mathrm{ml}$, with a sensitivity of $93.2 \%$ and a specificity of $76.5 \%$ for the diagnosis of VAP. The results of the current study demonstrated that measuring the sTREM-1 in the BALF and EVC were useful for diagnosing VAP following the result of a PCR assay in this population. However, a previous study revealed that measuring the sTREM-1 protein concentration in the mBALF did not discriminate patients with VAP from those without VAP following cardiac surgery in children (28). In the aforementioned study, mBALF was collected in a plastic container located at the centre of the exhaled portion of the ventilator tubing, which was far away from the patient's natural airway. Thus, the detection of sTREM-1 protein concentration and analysis of mBALF were inevitably affected by the bacterial colonization in the artificial airway. The difference between the two studies is that bronchoscopy was used for the BALF in the present study and the samples were obtained from deep within the pulmonary alveolus. The detection of sTREM-1 in BALF through the collection of BALF from the pulmonary alveolus was a more effective method as it was less likely to be influenced by an extrapulmonary infection. Additionally, the use of PCR for diagnosis of pneumonia has been demonstrated to be more sensitive in several studies $(29,30)$. In the present study, PCR was used for the pathogenic diagnosis of VAP and more pathogens were detected using that method compared with culture methods, reducing the rate of missed diagnoses.

One of the limitations of the current study is the lack of measurements of sTREM-1 concentrations prior to surgery to determine the patients' baseline levels. Another limitation is the lack of detection of viruses; the detection of viruses and bacteria by PCR will be performed in a future study.

To the best of our knowledge, the current study is the first to evaluate the diagnostic value of sTREM-1 and a PCR assay in the diagnosis of VAP following cardiac surgery. The detection of sTREM-1 protein concentration in the BALF and EVC samples may be useful for the diagnosis of VAP following 
paediatric heart surgery. The PCR assay defined the diagnosis and pathogens for VAP early in children undergoing cardiac surgery.

\section{Acknowledgements}

Not applicable.

\section{Funding}

The present study was supported by the National Natural Science Foundation of China (grant no. 81602818) and Science and Technology Commission of Shanghai Municipality (grant no. 15411967100).

\section{Availability of data and materials}

The datasets used and/or analyzed during the current study are available from the corresponding author on reasonable request.

\section{Authors' contributions}

CL, LZ and ZX designed the study. YL, QC and MZ collected the data. $\mathrm{CL}$ and $\mathrm{XG}$ analyzed the data. CL prepared the manuscript. CL, LZ and QC revised the manuscript. All authors read and approved the final manuscript

\section{Ethics approval and consent to participate}

The present study was approved by the Ethics Committee of Shanghai Jiaotong University School of Medicine and written informed consent was obtained from the patients' parents.

\section{Patient consent for publication}

Not applicable.

\section{Competing interests}

The authors declare that they have no competing interests.

\section{References}

1. Guardia Camí MT, Jordan García I and Urrea Ayala M: Nosocomial infections in pediatric patients following cardiac surgery. An Pediatr (Barc) 69: 34-38, 2008 (In Spanish).

2. Roeleveld PP, Guijt D, Kuijper EJ, Hazekamp MG, de Wilde RB and de Jonge E: Ventilator-associated pneumonia in children after cardiac surgery in The Netherlands. Intensive Care Med 37: $1656-1663,2011$.

3. Tarnok A and Schneider P: Pediatric cardiac surgery with cardiopulmonary bypass: Pathways contributing to transient systemic immune suppression. Shock 16 (Suppl 1): S24-S32, 2001.

4. Bassetti M, Taramasso L, Giacobbe DR and Pelosi P: Management of ventilator-associated pneumonia: Epidemiology, diagnosis and antimicrobial therapy. Expert Rev Anti Infect Ther 10: 585-596, 2012.

5. Melsen WG, Rovers MM, Groenwold RH, Bergmans DC, Camus C, Bauer TT, Hanisch EW, Klarin B, Koeman M, Krueger WA, et al: Attributable mortality of ventilator-associated pneumonia: A meta-analysis of individual patient data from randomised prevention studies. Lancet Infect Dis 13: 665-671, 2013.

6. Brix-Christensen V: The systemic inflammatory response after cardiac surgery with cardiopulmonary bypass in children. Acta Anaesthesiol Scand 45: 671-679, 2001.
7. Venkatachalam V, Hendley JO and Willson DF: The diagnostic dilemma of ventilator-associated pneumonia in critically ill children. Pediatr Crit Care Med 12: 286-296, 2011.

8. Fabregas N, Ewig S, Torres A, El-Ebiary M, Ramirez J, de La Bellacasa JP, Bauer T and Cabello H: Clinical diagnosis of ventilator associated pneumonia revisited: Comparative validation using immediate post-mortem lung biopsies. Thorax 54: 867-873, 1999.

9. Pontrelli G, De Crescenzo F, Buzzetti R, Calò Carducci F, Jenkner A, Amodio D, De Luca M, Chiurchiu S, Davies EH, Simonetti A, et al: Diagnostic value of soluble triggering receptor expressed on myeloid cells in paediatric sepsis: A systematic review. Ital J Pediatr 42: 44, 2016.

10. Mazzucchelli I, Garofoli F, Ciardelli L, Borghesi A, Tzialla C, Di Comite A, Angelini M, Tinelli C, Merlini G and Stronati M: Diagnostic performance of triggering receptor expressed on myeloid cells-1 and CD64 index as markers of sepsis in preterm newborns. Pediatr Crit Care Med 14: 178-182, 2013.

11. Palazzo SJ, Simpson T and Schnapp LM: Triggering receptor expressed on myeloid cells type 1 as a potential therapeutic target in sepsis. Dimens Crit Care Nurs 31: 1-6, 2012.

12. Determann RM, Millo JL, Gibot S, Korevaar JC, Vroom MB, van der Poll T, Garrard CS and Schultz MJ: Serial changes in soluble triggering receptor expressed on myeloid cells in the lung during development of ventilator-associated pneumonia. Intensive Care Med 31: 1495-1500, 2005.

13. Matsuno AK and Carlotti AP: Role of soluble triggering receptor expressed on myeloid cells-1 for diagnosing ventilator-associated pneumonia after cardiac surgery: An observational study. BMC Cardiovasc Disord 13: 107, 2013

14. Yu Y, Zhu C, Liu C, Gao Y, Yin R and Cao J: Diagnostic performance of soluble triggering receptor expressed on myeloid cells-1 in ventilator-associated pneumonia of patients with ischemic stroke. Can J Infect Dis Med Microbiol 2017: 9513690, 2017.

15. Murphy J, O' Rourke S, Corcoran M, O' Sullivan N, Cunney R and Drew R: Evaluation of the clinical utility of a real-time PCR assay for the diagnosis of streptococcus pneumoniae bacteremia in children: A retrospective diagnostic accuracy study. Pediatr Infect Dis J 37: 153-156, 2018.

16. Chen YS, Liu PY, Huang YF, Chen CS, Chiu LH, Huang NY, Hsieh KS and Chen YS: Comparison of diagnostic tools with multiplex polymerase chain reaction for pediatric lower respiratory tract infection: A single center study. J Microbiol Immunol Infect 46: 413-418, 2013.

17. Ngo TT, Hoang VT, Tran TL, Trinh VS, Tran T, Dao TQ, Phan QH, Meyer CG and Le HS: Clinical utility of an optimised multiplex real-time PCR assay for the identification of pathogens causing sepsis in vietnamese patients. Int J Infect Dis 67: 122-128, 2018.

18. Jordana-Lluch E, Gimenez M, Quesada MD, Ausina V and Martro E: Improving the diagnosis of bloodstream infections: PCR coupled with mass spectrometry. Biomed Res Int 2014: 501214, 2014

19. Vutukuru MR, Sharma DK, Ragavendar MS, Schmolke S, Huang Y, Gumbrecht W and Mitra N: A rapid, highly sensitive and culture-free detection of pathogens from blood by positive enrichment. J Microbiol Methods 131: 105-109, 2016.

20. Du Rand IA, Barber PV, Goldring J, Lewis RA, Mandal S, Munavvar M, Rintoul RC, Shah PL, Singh S, Slade MG, et al: British thoracic society guideline for advanced diagnostic and therapeutic flexible bronchoscopy in adults. Thorax 66 (Suppl 3): iiil-iii21, 2011.

21. Balakrishnan G, Aitchison T, Hallworth D and Morton NS: Prospective evaluation of the Paediatric Risk of Mortality (PRISM) score. Arch Dis Child 67: 196-200, 1992.

22. Mildh L, Pettilä V, Sairanen H and Rautiainen P: Predictive value of paediatric risk of mortality score and risk adjustment for congenital heart surgery score after paediatric open-heart surgery. Interact Cardiovasc Thorac Surg 6: 628-631, 2007.

23. Lauzier F, Ruest A, Cook D, Dodek P, Albert M, Shorr AF Day A, Jiang X and Heyland D: The value of pretest probability and modified clinical pulmonary infection score to diagnose ventilator-associated pneumonia. J Crit Care 23: 50-57, 2008.

24. Salehifar E, Tavakolian Arjmand S, Aliyali M, Abedi S, Sharifpour A, Alipour A, Ala S, Eslami G, Bozorgi F, Mahdavi MR and Walley KR: Role of C-reactive protein and tumor necrosis factor-alpha in differentiating between ventilator-associated pneumonia and systemic inflammatory response syndrome without infectious etiology. Tanaffos 15: 205-212, 2016. 
25. Klompas M, Branson R, Eichenwald EC, Greene LR, Howell MD, Lee G, Magill SS, Maragakis LL, Priebe GP, Speck K, et al: Strategies to prevent ventilator-associated pneumonia in acute care hospitals: 2014 update. Infect Control Hosp Epidemiol 35 (Suppl 2): S133-S154, 2014.

26. Chiu YH, Chen TJ, Chen CT and Lu CC: Positive blood cultures in pediatric emergency department patients: Epidemiological and clinical characteristics. Acta Paediatr Taiwan 46: 11-16, 2005.

27. Shin JH, Song SA, Kim MN, Lee NY, Kim EC, Kim S, Koo SH Ryoo NH, Kim JS and Cho JH: Comprehensive analysis of blood culture performed at nine university hospitals in Korea. Korean J Lab Med 31: 101-106, 2011.

28. Schindler MB and Cox PN: A simple method of bronchoalveolar lavage. Anaesth Intensive Care 22: 66-68, 1994.
29. Siow WT, Koay ES, Lee CK, Lee HK, Ong V, Ngerng WJ, Lim HF, Tan A, Tang JW and Phua J: The use of polymerase chain reaction amplification for the detection of viruses and bacteria in severe community-acquired pneumonia. Respiration 92: 286-294, 2016

30. CarrolED,MankhamboLA,GuiverM,BandaDL;IPDStudyGroup, Denis B, Dove W, Jeffers G, Molyneux EM, Molyneux ME, et al: PCR improves diagnostic yield from lung aspiration in Malawian children with radiologically confirmed pneumonia. PLoS One 6: e21042, 2011.

(7)(9) This work is licensed under a Creative Commons Attribution-NonCommercial-NoDerivatives 4.0 International (CC BY-NC-ND 4.0) License. 